\title{
ESTUDIO DE ALTERNATIVAS PARA LA RECUPERACION DE AGUAS RESIDUALES EN EL PROCESO DE OBTENCIÓN DE ACEITE DE PALMA EN LA PLANTA DE BENEFICIO DE GUAICARAMO S.A. META
}

Por:

Emilio Vera Duarte ${ }^{1}$, Luz Marina Herrera² ${ }^{2}$ Emerson Ordóñez Pabón ${ }^{3}$; Guido Alberto Sierra Ramírez. ${ }^{4}$

\section{RESUMEN}

En la planta de Guaicaramo, para el proceso de obtención del aceite de palma, las calderas producen la mayor demanda de agua utilizada en el proceso, aproximadamente $96000 \mathrm{~m} 3$ por año, este vapor al ser utilizado en este proceso genera condensados, que al reutilizarlos disminuiría la demanda de agua proveniente de pozos profundos y del río, por lo que este estudio plantea el diseño de un sistema de recuperación de los condensados que cumplan según parámetros determinados por la ABMA (American Boiler Manufacture Asociation) para nuevamente alimentar a la caldera.

También se analizaron los efluentes finales del sistema de tratamiento (EFST) de diferentes partes del proceso que llegan a una piscina de lodos para su recuperación, para estas se propuso un diseño experimental utilizando materias primas de residuo de la empresa (cuescos de palma, fibra y tusa) y un solo tratamiento tradicional (Filtración o floculación o sedimentación) y mezclas con agua para buscar su posterior utilización como agua de alimentación de calderas.

Palabras Claves: diseño experimental, eficiencia de calderas, recuperación de aguas residuales, recuperación de condensados.

\section{ABSTRACT}

In the plant of Guaicaramo, for the process of obtaining of the palm oil, the boilers produce the greater demand of water used in the process, approximately $96000 \mathrm{~m}^{3}$ per year, this steam to the being used in this process generates condensed, that when reusing them would diminish the demand of originating water of deep wells and the river, reason why this study put forward the design of a recovery system of the condensed ones that fulfill according to parameters determined by ABMA (American Boiler Manufacture Asociation) to feed the boiler again. Also the final efluentes of the system of treatment (EFST) of different parts from the process were analyzed that arrive at a mud swimming pool for their recovery, for these seted out an experimental design using raw materials of remainder of the company (cuescos of palm, fiber and tusa) and a single traditional treatment (Filtration or flocculation or sedimentation) and mixtures with water to look for their later use like water of feeding of boilers.

Keywords: Design experimental, efficiency of boilers, recovery of residual waters, recovery of having condensed.

\section{INTRODUCCION}

Colombia se encuentra posicionado como el primer productor de Palma (Elaeis guineensis) en América Latina y cuarto en el mundo; particularmente, el departamento del Meta tiene una participación del $31 \%$ del total nacional ocupando el primer lugar de área bruta sembrada con la oleaginosa contando con 47.525 hectáreas que registran una producción de 133.622 toneladas año, con un rendimiento de 2.8 toneladas de aceite crudo por hectárea ${ }^{5}$. Para la extracción de aceite de palma en la planta beneficio GUAICARAMO S.A., en los diferentes procesos que debe sufrir el fruto de la palma para la obtención del aceite es necesario el uso de vapor a altas condiciones de presión y temperatura. En la actualidad algunas plantas extractoras o de beneficio realizan procesos de esterilización, desfrutado, prensado y otros, en donde la demanda en cuanto a la producción de vapor es cuantiosa. De la cantidad de agua que se utiliza para la producción del aceite, el $70 \%$ es para producir el vapor necesario para el proceso de extracción, siendo una cantidad considerable incurriendo en costos ocasionados por el trámite, concesión de aguas con las

\footnotetext{
1 Ing. Mecánico. Luis Emilio Vera Duarte, Msc. Profesor investigador Grupo Fluter. UFPS. emilioveradua@gmail.com

2 Ing. Quimica Luz Marina Herrera León, Esp. Estadística. Est. Msc Estadística, Profesor investigador Grupo Fluter.

UFPS. luzherr@gmail.com

3 Ing. Mecánico. Emerson Ordoñez Pabón, Ingeniero Investigador. UFPS. Emerson_or@yahoo.com

4 Ing. Químico. Guido Alberto Sierra Ramírez. CENIPALMA.guidosierra@gmail.com

5 www.gobernaciondelmeta.gov.co/websiteg/index.php, septiembre, 2005.
} 


\section{ESTUDIO DE ALTERNATIVAS PARA LA RECUPERACION DE AGUAS RESIDUALES EN EL PROCESO DE OBTENCIÓN DE ACEITE DE PALMA EN LA PLANTA DE BENEFICIO DE GUAICARAMO S.A. META}

corporaciones autónomas regionales, y tratamientos para su utilización. Con el fin de aportar una solución a este problema y reducir este consumo se plantea la recirculación de los condensados generados en la planta diseñando un sistema de recirculación, el cual permitirá que el proceso mejore en eficiencia logrando una optimización de la planta e igualmente reducir costos de químicos en el tratamiento de agua. Para ello se inicia haciendo un balance de masa de las pérdidas de condensado, se caracterizan y se compararan con los datos estándar ABMA. En el diseño se eligen los componentes necesarios del sistema de recirculación, los parámetros de diseño óptimos del sistema se escogen de acuerdo a catálogos y manuales de proveedores y se realiza un análisis económico en cuanto a la reducción de químicos y agua en la planta. En el balance de masa de la caldera se tuvo en cuenta la cantidad de químico utilizado, Se elabora el plano de construcción y montaje del sistema.

También se estudiaron alternativas para recuperar el agua residual de las calderas, se aplicaron separadamente cuatro tratamientos convencionales 5 ampliamente referenciados en la literatura como sedimentación, filtrado, floculación y oxidación con inyección de aire, y establecer la posibilidad de recircularlos al proceso como agua de alimentación a las calderas, determinando paralelamente la reducción en el impacto ambiental y los costos generados por esta práctica y la posible implementación de alguno de los tratamientos de algunos de los tratamientos antes mencionados.

El estudio esta enmarcado dentro de los artículos 5 y 15 de la ley 373 de 1997, la cual establece el programa para el uso eficiente del agua potable.

GANANCIAS QUE SE OBTIENEN CON LA RECIRCULACIÓN DE LOS CONDENSADOS. La recirculación de condensados beneficia aspectos como eficiencia de las calderas, eficiencia del proceso, reducción del consumo de reactivos químicos, ahorro en energía, y disminución del consumo de agua de la planta.

\section{METODOLOGIA Y EQUIPOS}

Para el análisis de condensados se realizó una investigación descriptiva y comparada, tomando muestras aleatorias en los distintos puntos de producción y analizando sus propiedades físico-químicas, y luego comparándolas con los parámetros de la tabla 1dados por la ABMA para agua de calderas.

Los análisis realizados a los condensados fueron: $\mathrm{pH}$, dureza, oxígeno, alcalinidad, sulfitos, fosfatos, sílice, e hierro de muestras puntuales tomadas cada 2 horas por 3 veces al día en los puntos seleccionados.

Tabla 1. Parámetros Físico- Químicos exigidos para agua de caldera.

\begin{tabular}{|c|c|c|c|c|c|c|}
\hline ABMA & $\mathrm{pH}$ & $\begin{array}{c}\text { Dureza } \\
\text { ppm }\end{array}$ & $\begin{array}{c}\text { Oxígeno } \\
\text { ppm } \mathrm{O}_{2}\end{array}$ & $\begin{array}{c}\text { Hierro } \\
\text { ppm Fe }\end{array}$ & $\begin{array}{c}\text { Sílice } \\
\text { ppm } \mathrm{SiO}_{2}\end{array}$ & $\begin{array}{c}\text { Alcalinidad M } \\
\text { ppm } \mathrm{CaCO}_{3}\end{array}$ \\
\hline Parámetros & $<\mathbf{1 0}$ & $\mathbf{0}$ & $<\mathbf{0}$ & $<\mathbf{0 . 1}$ & $\mathbf{2 - 8}$ & $\mathbf{4 4 . 0 1}$ \\
\hline
\end{tabular}

Evaluación de procesos de tratamiento sobre las mezclas efluente - agua: Para el desarrollo del trabajo, se evaluó la respuesta de cuatro mezclas de EFST-Agua a cuatro tratamientos convencionales, con el objetivo de establecer la posibilidad de reutilizar una de estas mezclas como agua de alimentación a la caldera. Para el estudio de alternativas de recuperación se utilizo un diseño experimental como se muestra en la figura 1, en esta se muestra para cada tratamiento el porcentaje de mezcla efluente-agua utilizado los materiales utilizados para el proceso de filtración y floculación (residuos del proceso de extracción de aceite), al igual que las aperturas utilizadas para el proceso de oxidación. 


\section{ESTUDIO DE ALTERNATIVAS PARA LA RECUPERACION DE AGUAS RESIDUALES EN EL PROCESO DE OBTENCIÓN DE ACEITE DE PALMA EN LA PLANTA DE BENEFICIO DE GUAICARAMO S.A. META}

Figura 1. Diseño experimental para los tratamientos propuestos

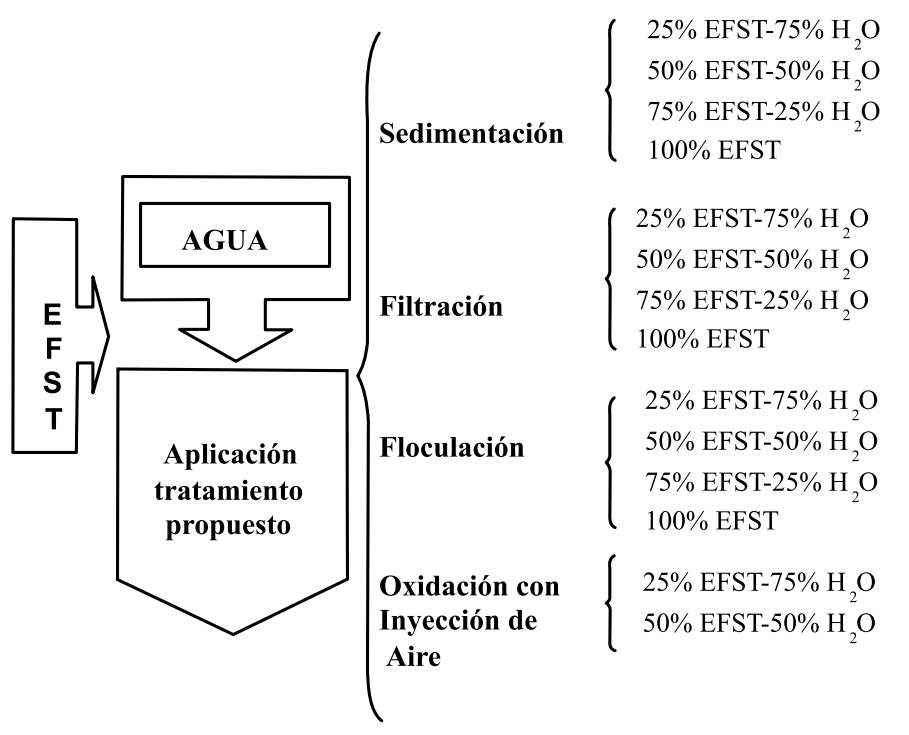

Sedimentación. Se diseñó un sedimentador piloto para un volumen de 20 litros de efluente y un tiempo de residencia de 3 horas para separar la mayor cantidad de sólidos posible; Una vez puesto en marcha, se tomaron muestras de $50 \mathrm{ml}$ cada 30 minutos para las diferentes concentraciones de efluente. Cada muestra fue sometida a la caracterización mencionada anteriormente para los condensados. Las concentraciones de efluente utilizado fueron: $5,50,75,100 \%$, se realizaron 9 repeticiones para un total de 36 ensayos.

Floculación. Se realizó el proceso de floculación utilizando sulfato de aluminio (Al2SO4) e hidróxido de sodio $(\mathrm{NaOH})$ para controlar el $\mathrm{pH}$. Se obtuvieron muestras de forma similar a la del tratamiento de sedimentación analizando sus características físico-químicas. También se utilizó otro floculante recomendado en la literatura es el (E615) (dosis $6 \mathrm{ml}$ ), esta compuesto por hidroxicloruro (dosis 7.5, 10.5 gr) y sulfato de aluminio (dosis 10,15 gr), este fue evaluado de la misma manera. La Concentración de efluente utilizada fue: $25,50,75,100 \%$, se realizaron 9 repeticiones para un total de 72 ensayos.

Filtrado: La literatura reporta la utilización de filtros de diferente tipo para el tratamiento del efluente residual en plantas de beneficio antes del tratamiento anaeróbico de las lagunas, dentro de los que se destacan los lechos de materiales orgánicos (fibra, tusa) y sus cenizas. Por esta razón se evaluaron estos materiales por separado y en serie para establecer su efecto sobre el efluente final de las lagunas de estabilización, realizando el proceso de filtrado en el laboratorio con una bomba dosificadora para agilizar el proceso. Se realizó la correspondiente caracterización físico-química de la muestra final en cada uno de los dos casos. La concentración de efluente utilizada fue: $25,50,75$, $100 \%$, se realizaron 9 repeticiones para un total de 72 ensayos

Oxidación con inyección de aire. Al tanque sedimentador se le agrego por la parte inferior aire a presión, el tiempo de residencia y el procedimiento de toma y acumulación de la muestra fueron similares a los utilizados en el tratamiento de sedimentación. La concentración de efluente utilizada fue: $25,50,75$, $100 \%$, se realizaron 9 repeticiones para un total de 36 ensayos

\section{EFICIENCIA DE LA CALDERA:}

A continuación se describe detalladamente como se realizo cada balance en cuanto a las mediciones de fibra, agua, cascarilla y humedad.

En primera instancia se necesita saber cuanto es el total de fruto procesado diariamente y esto se logra por medio del programa de producción en el cual aparece registrado cuanto es el fruto que entra a la plantación por día, cuanto fruto se procesa diariamente, cuanta cantidad de aceite se produjo, cuanto se despacho, entre otros ítems.

Las horas efectivas al igual que el número de vagonetas procesadas quedan registradas diariamente en las planillas por los operarios, también estos son 


\section{ESTUDIO DE ALTERNATIVAS PARA LA RECUPERACION DE AGUAS RESIDUALES EN EL PROCESO DE OBTENCIÓN DE ACEITE DE PALMA EN LA PLANTA DE BENEFICIO DE GUAICARAMO S.A. META}

proporcionados por el programa de producción. El peso promedio por vagoneta resulta de la siguiente manera:

$$
\text { Peso promediovagoneta }=\frac{\text { Fruto procesado/ día }}{\text { Número de vagonetas } / \text { día }}
$$

Los aforos de fibra se realizaron por un periodo de 5 días durante 25 minutos por cada ensayo, aunque este tiempo puede variar lo que permitiría obtener un dato más confiable. Para esta medición se necesito tener disponibilidad de un zorrillo vacío y previamente pesado, en donde se vertería toda la cantidad de fibra que se produjera en ese momento, donde se dio la orden al operario de cerrar la compuerta de la alimentación a la caldera, alimentándola de forma manual con cascarilla durante la prueba para impedir que se bajara la producción de vapor y posteriormente la presión. Después de terminado el ensayo se procede a pesar nuevamente el zorrillo para saber cuantas toneladas de fibra se produjeron durante este lapso de tiempo. El total de fibra producida por día se deduce de:

Fibra producida $/$ día $=\frac{\text { Ton de fibra medida } * \text { Horas efectivas }}{\text { Tiempo medición }(h)}$

(Ton)

La formula que expresa el porcentaje de fibra producida con respecto al total de RFF (racimo de fruta fresca) procesados esta dada por

$\%$ fibra producida/RFF $=\frac{\text { Fibra producida/día }}{\text { Fruto procesado/día }} * 100$

La fibra desperdiciada deja de ser aprovechada como combustible que se produce cuando se cierra el paso de alimentación hacia las calderas ya sea por diferentes contratiempos como paradas, poca demanda de vapor, la cantidad de fruto procesado entre otros, rebosando los silos de almacenamiento.

El total de fibra pérdida en la planta esta dada por

Fibra pérdidal día $=\frac{\text { Ton de fibra pérdida medida* Horas efectivas }}{\text { Tiempo medición }(h)}$

(Ton)

Para determinar la humedad que contiene la fibra, el ensayo se realizo por método gravimétrico, utilizando una cápsula previamente pesada, tomando una muestra de fibra de $10 \mathrm{~g}$. Se procedió a llevar el conjunto al horno eléctrico por un periodo de dos horas a una temperatura de $105^{\circ} \mathrm{C}$, (procedimiento seguido del manual de laboratorio de Cenipalma). Después de haberse cumplido el tiempo se retiró la muestra del horno dejándola en reposo durante un minuto, Pesando nuevamente se procede a calcular el contenido de humedad por:

$$
\% \text { humedad }=\frac{P_{t}-P_{f}}{P_{i}} * 100
$$

Donde: $P_{t}$ : Peso total, $P_{f}$ : Peso final $Y P_{i}$ : Peso inicial

Acerca del total de fibra disponible esta se obtiene de restar al total de fibra producida por día la cantidad desperdiciada y la humedad que esta contiene.

Total fibra disponible $=$ Fibra producida - Fibra pérdida -

$$
\text { Humedad (Ton / día) }
$$

Con respecto a la cantidad de fibra pérdida esta dada por:

$$
\% \text { fibrapérdidal } R F F=\frac{\text { Fibrapérdidal día }}{\text { Frutoprocesadol día }} * 100
$$

De la misma forma como se realizaron los aforos de fibra se hicieron los de cuesco seco (cascarilla). Las formulas empleadas para este caso son:

$$
\begin{gathered}
\text { Cuescoseco producido/ dia }=\frac{\text { Ton cuescoseco medido* Horas efectivas }}{\text { Tiempomedición }(h)} \text { (Ton) } \\
\% \text { cuescoseco producido/ RFF }=\frac{\text { cuescoseco producido/ día }}{\text { Frutoprocesado/ día }} * 100
\end{gathered}
$$

Para los aforos de cuesco húmedo se planteó realizarlo por un lapso de tiempo de 20-25 minutos, del material proveniente de los hidrociclones. Los cálculos de este material se obtienen de:

Cuesco humedo/dia $=\frac{\text { Ton de cuescohumedomedido*Horas efectivas }}{\text { Tiempomedición }(h)}$ (Ton)
$\%$ cuesco humedo producido $/$ RFF $=\frac{\text { cuesco humedo producido / día }}{\text { Fruto procesado / día }} * 100$

La cantidad de cuesco perdido se produce cuando se cierra el paso de alimentación hacia las calderas. Los datos de las mediciones de la pérdida de cuesco seco y húmedo fueron proporcionados en la báscula para saber la cantidad que se estaba desperdiciando.

Cuesco perdido $/$ día $=\frac{\text { Ton de cuesco perdido * Horas efectivas }}{\text { Tiempo medición }(h)}$ (Ton)

Para calcular la humedad de la cascarilla se realizó por el mismo método que para la fibra.

$$
\% \text { humedad }=\frac{P_{t}-P_{f}}{P_{i}} * 100
$$




\section{ESTUDIO DE ALTERNATIVAS PARA LA RECUPERACION DE AGUAS RESIDUALES EN EL PROCESO DE OBTENCIÓN DE ACEITE DE PALMA EN LA PLANTA DE BENEFICIO DE GUAICARAMO S.A. META}

donde: $\quad P_{t}=$ Peso total $\quad P_{f}=$ Peso final $P_{i}=$ Peso inicial

Para calcular el total de cuesco disponible en la planta dado en toneladas por día se tiene por:

\section{Cuesco disponible $=$ Cuesco seco + cuesco húmedo - perdidas de cuesco - humedad}

La pérdida de cuesco esta dada por:

$\%$ cuesco perdido $/$ RFF $=\frac{\text { Cuesco perdido } / \text { día }}{\text { Fruto procesado } / \text { día }} * 100$

La capacidad calorífica fue obtenida por Cenipalma. La energía disponible se obtiene por la ecuación:

Para el caso es: $\quad E d=m \cdot C p \cdot \Delta T$

$$
E d=m_{\text {fibradisponible }} \cdot C p_{\text {fibra }} . \Delta T+m_{\text {cuescodisponible. }} . C p_{\text {cuesco }} . \Delta T
$$

La cantidad de agua utilizada en las calderas se determina por método volumétrico, la cantidad de vapor producido se obtiene por:

Cantidad de vapor $=Q \cdot H_{\text {bomba } 1}+Q \cdot H_{\text {bomba } 2}$

(Ton / día)

El caudal (Q) esta dado en $\mathrm{m}^{3} / \mathrm{h}$ y el tiempo (h) esta dado en horas indicando por los hodómetros de cuanto tiempo trabajaron las bombas durante el día.

La energía consumida esta dada por la expresión:

$$
E c=\left(\frac{\dot{m} \cdot C p \cdot \Delta T}{C p_{\text {agua }}}\right)+\left(\frac{\dot{m} \cdot C p \cdot \Delta h}{C p_{\text {agua }}}\right)
$$

$(k c a l / d i a)_{* * * * * * *}$

La eficiencia de una caldera esta dado por la ecuación:

$$
\eta=\left(\frac{E_{\text {disponible }}}{E_{\text {consumida }}}\right) \cdot 100
$$

Tomada del manual de cálculos de Cenipalma6.

\section{RESULTADOS}

GENERACIÓN TOTAL DE CONDENSADOS. En el cuadro 1 se encuentran los resultados promedios para cada punto de generación, donde se puede observar que cantidad que se produce por hora, por día y por tonelada de racimo de fruta fresca (Ton RFF), en cada punto y se observa que es considerable la producción de condensado $1.59\left(\mathrm{~m}^{3} / \mathrm{h}\right)$, por lo que se benefició la planta al recuperar estos condensados que cumplían con las condiciones físico-químicas para ser reutilizados como agua de caldera.

Cuadro 1. Flujo de condensados en la planta de beneficio

\begin{tabular}{|l|c|c|c|c|}
\hline \multicolumn{1}{|c|}{ Punto } & $\begin{array}{c}\text { Flujo } \\
\left(\mathbf{m}^{\mathbf{3}} / \mathbf{h}\right)\end{array}$ & Flujo $\left(\mathbf{m}^{\mathbf{3}} / \mathbf{d i ́ a}\right)$ & $\mathbf{m}^{\mathbf{3}} /$ Ton RFF & $\begin{array}{c}\text { Te mperatura } \\
\left({ }^{\circ} \mathbf{C}\right)\end{array}$ \\
\hline Distribuidor Vapor & 0,140161 & 2,312662 & 0,0090739 & 96,22 \\
\hline Sedimentador de aceite & 0,017280 & 0,285120 & 0,00223943 & 88,00 \\
\hline Silo secado almendra & 0,310000 & 54,115000 & 0,04030715 & 93,52 \\
\hline Silos secado & 0,335421 & 5,534443 & 0,02165618 & 96,44 \\
\hline Sistema secado al vació & 0,014920 & 0,246180 & 0,00171199 & 89,84 \\
\hline Tanque de crudos & 0,203860 & 3,363690 & 0,02650650 & 92,22 \\
\hline Tanque de lodos & 0,062721 & 1,034896 & 0,00402056 & 87,62 \\
\hline Tanque almacenamiento \# 1 & 0,132276 & 2,182555 & 0,00861595 & 93,30 \\
\hline Tanque de almacenamiento \#4 & 0,244761 & 4,038555 & 0,01626073 & 93,48 \\
\hline Transportador de torta & 0,130464 & 2,152656 & 0,00848166 & 92,00 \\
\hline Total & $\mathbf{1 , 5 9 1 8 6 4}$ & $\mathbf{2 6 , 2 6 5 8}$ & $\mathbf{0 , 1 3 8 8 7 4}$ & $\mathbf{9 2 , 2 6}$ \\
\hline
\end{tabular}

CARACTERIZACIÓN DE CONDENSADOS. En el cuadro 2 se muestran los resultados de la caracterización: pH, Dureza, Oxigeno disuelto, Hierro, Sílice y Alcalinidad en los puntos determinados como generadores de condensados y se determinó que todos independientemente cumplían con la Norma ABMA

\begin{tabular}{|c|c|c|c|c|c|c|}
\hline Punto & $\mathrm{pH}$ & $\begin{array}{c}\text { Dureza } \\
\text { ppm }\end{array}$ & $\begin{array}{c}\text { Oxígeno disuelto } \\
{\mathrm{ppm} \mathrm{O}_{2}}\end{array}$ & $\begin{array}{l}\text { Hierro } \\
\text { ppm Fe }\end{array}$ & $\begin{array}{c}\text { Silice } \\
\text { ppm } \mathrm{SiO}_{2}\end{array}$ & $\begin{array}{c}\text { Alcalinidad M } \\
\text { ppm } \mathrm{CaCO}_{3}\end{array}$ \\
\hline Distribuidor Vapor & 9,01 & 0 & 0,00 & 0,02 & 3,2 & 100,98 \\
\hline Sedimentador & 6,90 & 0 & 0,00 & 0,00 & 5,68 & 31,37 \\
\hline Silos secado & 6,57 & 0 & 0,00 & 0,00 & 4,42 & 20,59 \\
\hline Silo secado almendra & 6,67 & 0 & 0,00 & 0,00 & 5,54 & 16,67 \\
\hline Tanque de almacenamiento \# 1 & 6,37 & 0 & 0,00 & 0,00 & 2,83 & 18,63 \\
\hline Tanque de almacenamiento \# 4 & 6,00 & 0 & 0,00 & 0,00 & 2,54 & 18,63 \\
\hline Tanque de alimentación & 5,75 & 0 & 0,00 & 0,67 & 8,40 & 14,71 \\
\hline Tanque de crudos & 6,03 & 0 & 0,12 & 0,00 & 1,23 & 14,71 \\
\hline Tanque de lodos & 6,00 & 0 & 0,12 & 0,01 & 0,49 & 26,47 \\
\hline Transportador de torta & 6,10 & 0 & 3,43 & 0,00 & 4,60 & 22,55 \\
\hline Sistema secado al vació & 6,58 & 0 & 0,00 & 0,00 & 1,13 & 14,71 \\
\hline Parámetros ABMA & $<10$ & $\mathbf{0}$ & $<0$ & $<0.1$ & $2-8$ & 44.01 \\
\hline
\end{tabular}

Cuadro 2. Caracterización de condensados generados en la planta.

Al comparar las características obtenidas de cada punto con los parámetros establecidos, se observa que en general todos los condensados evaluados pueden ser recirculados sin ningún problema como agua de alimentación a las calderas.

\section{EFICIENCIA DE LA CALDERA}

El cuadro 3 muestra que la eficiencia de las calderas de la planta Beneficio se encontraban por debajo de los parámetros recomendados por la ABMA, cuyos

6 CENIPALMA, Algunos aspectos del procesamiento de aceite de palma. Modulo II Calidad del Aceite. Modulo III Esterilización. Modulo IV Clarificación. Modulo VI tratamiento de efluentes. Bucaramanga. Diciembre 2 y 3 de 1993. 
valores están entre el 70 y $75 \%$, para una caldera en optimas condiciones debe tener como mínimo del $80 \%$, por lo que se determinó la necesidad de construir un sistema de recirculación de condensado para mejor la eficiencia de las calderas.

Cuadro 3. Balance de las calderas de Guaicaramo S.A.

\begin{tabular}{|l|c|c|c|c|c|}
\hline \multicolumn{1}{|c|}{ FECHA } & $30 / 08 / 05$ & $20 / 09 / 05$ & $21 / 09 / 05$ & $22 / 09 / 05$ & $23 / 09 / 05$ \\
\hline Fibra disponible (Ton/día) & 23,71 & 22,89 & 21,42 & 16,37 & 21,48 \\
\hline Cuesco disponible (Ton/día) & 16,177 & 10,322 & 12,582 & 20,959 & 13,190 \\
\hline Energía disponible (kCal/día) & 137.342 .89 & 112.476 .98 & 116.366 .27 & 132.025 .15 & 118.872 .31 \\
\hline Energía consumida (kCal/día) & 96.501 .665 & 57.255 .089 & 71.599 .160 & 79.435 .115 & 94.356 .832 \\
\hline EFICIENCIA CALDERAS $\left(\mathrm{n}_{\mathrm{c}}\right)$ & $70,26 \%$ & $50,90 \%$ & $61,53 \%$ & $60,17 \%$ & $74,38 \%$ \\
\hline
\end{tabular}

DISEÑO DEL SISTEMA DE RECIRCULACIÓN DE CONDENSADOS. Este diseño ayudó a mejorar la eficiencia del sistema, para este tipo de fluidos se recomienda utilizar bombas del tipo PPC (Power pump condensate), utilizando vapor como fuerza motriz, la cual disminuye la cantidad de revaporizado; no es recomendable usar bombas eléctricas debido a que la mezcla de agua revaporizado se tendría que enfriar por debajo de los $86^{\circ} \mathrm{C}-212^{\circ} \mathrm{F}$ para evitar la cavitación de la bomba.

La instalación de bomba PPC en el sistema de recirculación de condensados se muestra en la figura 6, los códigos de los accesorios son: 1 - Bomba de condensados PPC 1 1/2" con válvulas de cheque en acero inoxidable, 2- Válvula M1OS2 1 1/2" NPT, 3- Filtro IT 1 1/2" NPT, 4- Válvula M1OS2 1 1/2" NPT, 5Trampa termodinámica TD42L 1/2" NPT, 6- Válvula M1OS2 1/2" NPT, 7- Rompedor de vació VB14 1/2" NPT, 8- Eliminador de aire para líquidos 13WS 3/4" NPT y 9- Válvula cheque DCV3 1 1/2" ANSI 150

Figura 6. Instalación bomba PPC

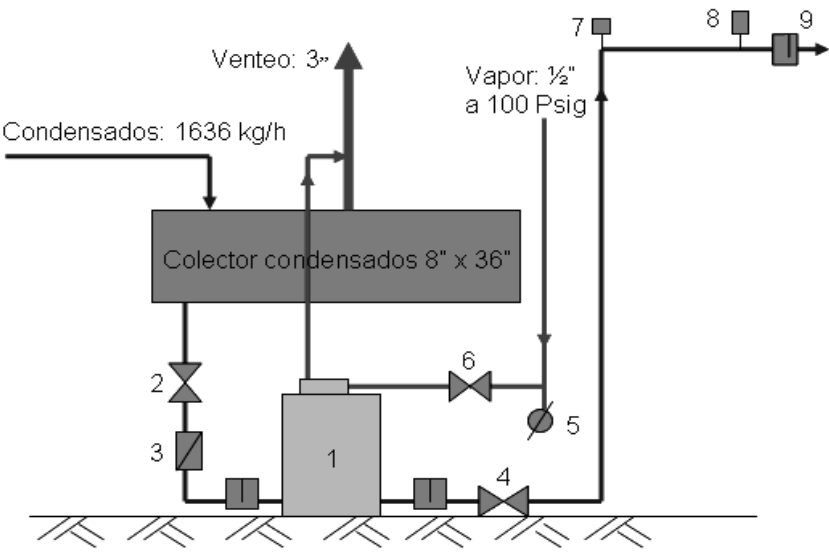

EVALUACIÓN DE TRATAMIENTOS. De todos los ensayos propuestos en el diseño experimental la mezcla que arrojó los mejores resultados fue la de 25\% efluente y $75 \%$ agua. En esta mezcla se mejoraron las propiedades físico-químicas de los efluentes. Los resultados se compararon con la norma estándar ABMA, aunque se estableció que ningún tratamiento cumplió con la norma recomendada para aguas de caldera en dureza, alcalinidad y contenido de sílice

Como en todos los tratamientos propuestos en el diseño experimental es necesario mejorar la dureza, la alcalinidad y contenido de sílice se presenta a continuación los costos necesarios para la utilización de agua residuales.

Costos Remoción de Propiedades del agua de alimentación. Remoción de dureza

Dosificación de acondicionador de dureza en las calderas:

Precio de dosificación por día:

$2.76 \mathrm{~kg} / \mathrm{dí} a$

Precio Tambor de $50 \mathrm{~kg}$. de acondi-

cionador de dureza:

$\$ 26.496$

$\$ 480.000$

Precio kg. de acondicionador de dureza: $\$ 9.600$

Este producto es apto para remover hasta $12 \mathrm{ppm}$ de dureza; por consiguiente para valores mayores se recomienda la instalación de un suavizador, cuyo costo depende de las ppm de dureza a remover.

El ciclo normal de regeneración de la resina es de 8 a 12 días y se realiza con sal industrial, la cantidad requerida de sal industrial para dicha regeneración es de $10 \mathrm{~kg} / \mathrm{ft}^{3}$.

Remoción de sílice. Para remover la cantidad de sílice del agua de alimentación se debe implementar un tren desmineralizador para remover todas las ppm de $\mathrm{SiO} 2$. Si se hace un buen manejo del suavizador y las propiedades del agua mejoran en ciertas cantidades el ciclo de regeneración se alarga, reduciendo en costos.

Remoción de alcalinidad. Para controlar la alcalinidad > 100 ppm de CaCO3 en el agua, se procede a añadirle ácidos (clorhídrico, sulfúrico, entre otros), con lo cual esta aumenta su acidez, lo que repercute directamente en el $\mathrm{pH}$; para controlar esta se le agrega cal teniendo el agua a ser nuevamente alcalina. 


\section{ESTUDIO DE ALTERNATIVAS PARA LA RECUPERACION DE AGUAS RESIDUALES EN EL PROCESO DE OBTENCIÓN \\ DE ACEITE DE PALMA EN LA PLANTA DE BENEFICIO DE GUAICARAMO S.A. META}

\section{CONCLUSIONES}

En el cuadro 1. se observa un potencial de recirculación de condensados de vapor como agua de alimentación a la caldera de aproximadamente $0.14 \mathrm{~m}^{3}$ / ton RFF.

La recirculación de condensados aumenta la eficiencia en las calderas hasta en un $5 \%$.

La mezcla $25 \%$ efluente y $75 \%$ agua que se muestra en el cuadro 4 en el tratamiento de floculación con E615 (6 ml/20 l), mejora significativamente las propiedades fisicoquímicas de las aguas residuales, aunque no alcanzaron valores similares a los del agua de la fuente utilizada para las calderas de la planta de beneficio.

Los tratamientos de floculación con $\mathrm{Al} 2 \mathrm{SO} 4$ e inyección de aire presentaron el mejor desempeño sobre las mezclas de EFST - agua evaluadas.

Se encontró que para la aplicación de un tratamiento independiente, si mejoran las condiciones físico-químicas de las aguas residuales del proceso, aunque no cumple con los requerimientos de la norma ABMA.

\section{RECOMENDACIONES}

Se recomienda realizar una revisión permanente de los diferentes dispositivos que generan los condensados como son los serpentines, válvulas y filtros, ya que cualquier problema que tenga uno de estos dispositivos se vera reflejado en la producción de la cantidad de condensados.

Se sugiere que antes de realizar cualquier tipo de ensayo con el agua del efluente final del sistema de tratamiento de las lagunas facultativas esta deben descolmatarse para así evitar posibles errores en los ensayos, ya que un problema que se observo durante la realización de los ensayos fue la cantidad de lodos presente en este tipo de agua, lo cual no permitió tener bien claro si la aplicación de alguno de estos tratamientos lograría tener propiedades adecuadas para su reutilización.

Se sugiere que el agua que se obtiene después de haber aplicado los tratamientos es apta para hacer diluciones o para ser utilizada en los hidrociclones, donde se reduciría el consumo de agua proveniente de las fuentes.

Cuadro 4. Resultados promedios del tratamiento (25\% EFST y $75 \%$ Agua)

\begin{tabular}{|c|c|c|c|c|c|c|c|c|c|c|c|c|}
\hline \multirow[b]{2}{*}{ Tratamiento } & \multicolumn{6}{|c|}{ Antes del tratamiento } & \multicolumn{6}{|c|}{ Después del tratamiento } \\
\hline & $\frac{T}{2}$ & 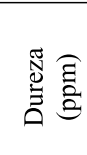 & 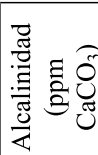 & 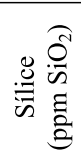 & 올 & 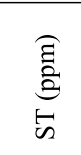 & 吾 & 总 & 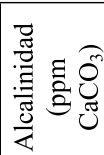 & 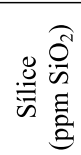 & 窇 & $\begin{array}{l}\widehat{\Xi} \\
\text { a् } \\
\text { t) }\end{array}$ \\
\hline Sedimentación & 8,24 & 666,2 & 426 & 140 & 0 & 1858 & 7,3 & 212,5 & 397,1 & 35,3 & 0 & 948 \\
\hline $\begin{array}{l}\text { Filtración } \\
\text { (Tusa + fibra) }\end{array}$ & 8,24 & 666,2 & 426 & 140 & 0 & 1374 & 7,83 & 625 & 536 & 116 & 0,1 & 1858 \\
\hline $\begin{array}{l}\text { Filtración lecho } \\
\text { de ceniza }\end{array}$ & 8,42 & 458,3 & 617 & 71 & 0 & 1810 & 8,91 & 406 & 654 & 93 & 0 & 1875 \\
\hline $\begin{array}{l}\text { Filtración doble } \\
\text { lecho de cenizas }\end{array}$ & 8,87 & 521 & 735,3 & 67,5 & 0 & 2563 & 9,94 & 525 & 529,4 & 50 & 0 & 1666 \\
\hline $\begin{array}{l}\text { Floculación }(10 \mathrm{~g} \\
\text { de } \mathrm{Al}{ }_{2} \mathrm{SO}_{4}+ \\
\mathrm{NaOH} 10 \mathrm{~g} / 20 \mathrm{l})\end{array}$ & 8,49 & 641,7 & 441 & 90 & 0,1 & 2251 & 9,45 & 50 & 764 & 9,16 & 0 & 1751 \\
\hline $\begin{array}{l}\text { Floculación (15 g } \\
\text { de } \mathrm{Al}{ }_{2} \mathrm{SO}_{4}+ \\
\mathrm{NaOH} 15 \mathrm{~g} / 20 \mathrm{l})\end{array}$ & 8,84 & 521 & 735 & 75 & 0 & 2667 & 9,65 & 250 & 809 & 10 & 0 & 2340 \\
\hline $\begin{array}{l}\text { Floculación con } \\
\text { E615 (6ml/20 l) }\end{array}$ & 7,33 & 583 & 1182 & 76,7 & 0 & 3006 & 7,92 & 440 & 701 & 26 & 0 & 2136 \\
\hline $\begin{array}{l}\text { Inyección aire } \\
\text { ( } 25 \% \text { apertura de } \\
\text { válvula) }\end{array}$ & 9,10 & 264 & 470,6 & 31,5 & 0 & 1543 & 9,12 & 244 & 433 & 26 & 0 & 1128 \\
\hline $\begin{array}{l}\text { Inyección aire } \\
\text { ( } 50 \% \text { apertura de } \\
\text { válvula) }\end{array}$ & 9,09 & 293 & 500 & 28 & 0 & 1689 & 9,06 & 237 & 419 & 26 & 0 & 1291 \\
\hline PARÁMETRO & $<\mathbf{1 0}$ & 0 & 44,1 & $4-8$ & 0,1 & $<\mathbf{5 0 0}$ & & & & & & \\
\hline
\end{tabular}




\section{ESTUDIO DE ALTERNATIVAS PARA LA RECUPERACION DE AGUAS RESIDUALES EN EL PROCESO DE OBTENCIÓN DE ACEITE DE PALMA EN LA PLANTA DE BENEFICIO DE GUAICARAMO S.A. META}

\section{AGRADECIMIENTOS.}

Los autores expresan su agradecimiento a los directivos de LA PLANTA DE BENEFICIO DE GUAICARAMO S.A. (META), por su financiación y apoyo logístico lo cual hizo posible la realización de este proyecto e igualmente a CENIPALMA. Por su aporte científico.

\section{BIBLIOGRAFIA}

[1] Anuário estadístico. Fedepalma. 2004.

A.N.Ma. 2000. Palm Oil developments, 30, págs 1-10.

[2] GARCIA NUÑEZ, JESUS ALBERTO. Boletín Técnico No 11. Manejo de Efluentes de Plantas Extractoras. Diseño de lagunas de estabilización. Diseño de lagunas de estabilización. Santafé de Bogotá D.C. Marzo 1997.

[3] CENIPALMA, Algunos aspectos del procesamiento de aceite de palma. Modulo II Calidad del Aceite. Modulo III Esterilización. Modulo IV Clarificación. Modulo VI tratamiento de efluentes. Bucaramanga. Diciembre 2 y 3 de 1993.

[4] TAJUDDIN, R. 2003. Oxygen enriched air using membrane for palm oil wastewater treatment. Songjklanakarin Journal Science Technology Vol. 24 Membrane Sci. \& Tech, págs 989-998

[5] JAMES. 2001. Destabilisation of oil - water emulsions and separation by dissolved air flotation. Water Research 36, págs 1503-1512

[6] AHMAD, ABDUL L. 2003. Water recycling from palm oil mill effluent (POME) using membrane technology. Desalination 157, págs 87 - 95.

[7] ZHOU, H. SMITH, D.W. 2002. Advanced technologies in water and wastewater treatment. J. Environ. Eng. Sci. 1, Págs $247-264$.
[8] SAVELSKI, M. BAGAJEWICZ, M. 2000. On the optimality conditions of water utilization systems in process plants with single contaminants. Chemical Engineering Science 55.Págs. 5035 - 5048.

[9] CENIPALMA, Manual de laboratorio Plantas de Beneficio primario para fruto de palma de aceite. 1998 - 1999.

[11] AVALLONE, Eugene A. and THEDORE BAUMEISTEIR. Manual del Ingeniero Mecánico. Quinta Edición.

[12] STREeTER, Víctor L., WYLIE, E. Benjamín. Mecánica de los Fluidos. Octava Edición. 1999.

[13] NOEL, Wambeck. Sinopsis del proceso de palma de aceite. Primera edición. 2005, Págs. 3 - 65 Manuales de EXRO Ltda. (Empresa que diseña y desarrolla soluciones integrales para el tratamiento de aguas) Catálogos de SPIRAX SARCO

[14] SAVELSKI, M. BAGAJEWICZ, M. 2000. On the optimality conditions of water utilization systems in process plants with single contaminants. Chemical Engineering Science 55.Págs. 5035 - 5048.

[15] AHMAD, ABDUL L. 2003. Water recycling from palm oil mill effluent (POME) using membrane technology. Desalination 157, págs 87 - 95.

[16] ZHOU, H. SMITH, D.W. 2002. Advanced technologies in water and wastewater treatment. J. Environ. Eng. Sci. 1, Págs $247-264$.

Recibido: 16 - Octubre - 2006

Aceptado: 19 - Junio - 2007 